\title{
A New Hotel Entrance Guard System
}

\author{
Fan Huan ${ }^{1, a}$, Jiahu Guo ${ }^{2, b}$, Zhang Sai ${ }^{3, c}$ \\ ${ }^{1}$ Collage of Electrical and Information Engineering, Anhui University of Science and Technology, \\ Huainan, Anhui, 232001, China \\ ${ }^{2}$ Collage of Electrical and Information Engineering, Anhui University of Science and Technology, \\ Huainan, Anhui, 232001, China \\ ${ }^{3}$ Collage of Electrical and Information Engineering, Anhui University of Science and Technology, \\ Huainan, Anhui, 232001, China \\ aemail: 630783168@qq.com, bemail: fanhuan2271@163.com, ${ }^{\text {cemail: } 1193039655 @ q q . c o m}$
}

Keywords: ZigBee; Hotel; Entrance Guard System

\begin{abstract}
At present, the development of the hotel entrance guard system tends to be more intelligent. The use of ZigBee wireless sensor networks and ARM embedded technology can solve communication problems for the hotel door terminal node with management server. Based on the Z-Stack, one of ZigBee standard protocol stack, we propose a new hotel access control system. The system selected of TI Company CC2530, adding a high-performance chip card reader MFRC522, has an IC card wireless identification and service management functions. Establish ZigBee wireless sensor networks can reduce the cost of a lot wiring brings and improve the appearance of the hotel. After testing, the program is suitable for access control systems to manage the hotel and the hotel staff can improve work efficiency and service levels.
\end{abstract}

\section{Introduction}

Access Control System is a digital management system for managing staff in and out. Since the continuous development of the hospitality industry, the demand for access control system is also undergoing significant changes [1] [2]. The management lock tends to intelligent not only improve the safety of Access Control System, but also is convenient to obtain staff information in and out [3] [4]. The access control system of traditional hotel with a large number wiring, each room and the master controller requires contact with the line, equipment installation and maintenance difficulties [5]. The contactless access control system uses contactless IC card which is easy to wear, lose data and less secure [6]. This paper designs a new type of hotel access control system, it contains the ZigBee wireless technology, ARM embedded technology and RFID technology [7]. In this system, all the rooms and floors can be centrally managed, the control center can display record of open the door real-time so that can improve the hotel's security. Its self-test function can reminder the management staff of fault information timely, and reduces the manpower to maintain [8] [9]. The lock terminal of this system uses low-power chips and extends the battery life.

\section{System Overall Structure}

This system mainly consists of two parts, one part is CC2530 locks terminal, the other part is Access Point (AP) which formed by ARM. Between the parts, ZigBee wireless network is used to communicate. System overall structure diagram is shown in figure 1.

AP is used to receive the news of the locks terminal and exchange data with hotel management server, including S3C2440A, CC2530 coordinator, node under voltage detecting module, node power management module, liquid crystal display module, Ethernet control module. Coordinator is used to establish the ZigBee wireless networks, process network information, and transmit information between terminal and AP. Nodes under voltage detecting module is used to test the power status of AP. Ethernet control module can be used for connecting management server, and is connected to the management server through the Ethernet. 
Door Lock Terminal is used for data exchange with the AP, controlling the switch of door lock, and providing the service interface. It is mainly including CC2530, infrared detecting circuit, voltage detection circuit, key circuit, motor drive circuit, indicating circuit, RF card read/write module. CC2530 is MCU. Infrared detecting circuit is used to monitor whether there is a card, and will start the card reader if a card is existence. Card reader is used to receive and process the information of the card. Locks terminal adopts $6 \mathrm{~V}$ battery powers, and when the supply voltage of voltage detecting circuit detects system is in a low voltage, system alarms. Key circuit provides service for the user, once the button is pressed, guest room management center will arrange the corresponding service personnel to provide services. Motor drive circuit is connected to the lock body, which is used to control the door lock of the door. Indicating circuit has sound and light indications, which prompt the user whether swiping card or key is successful.

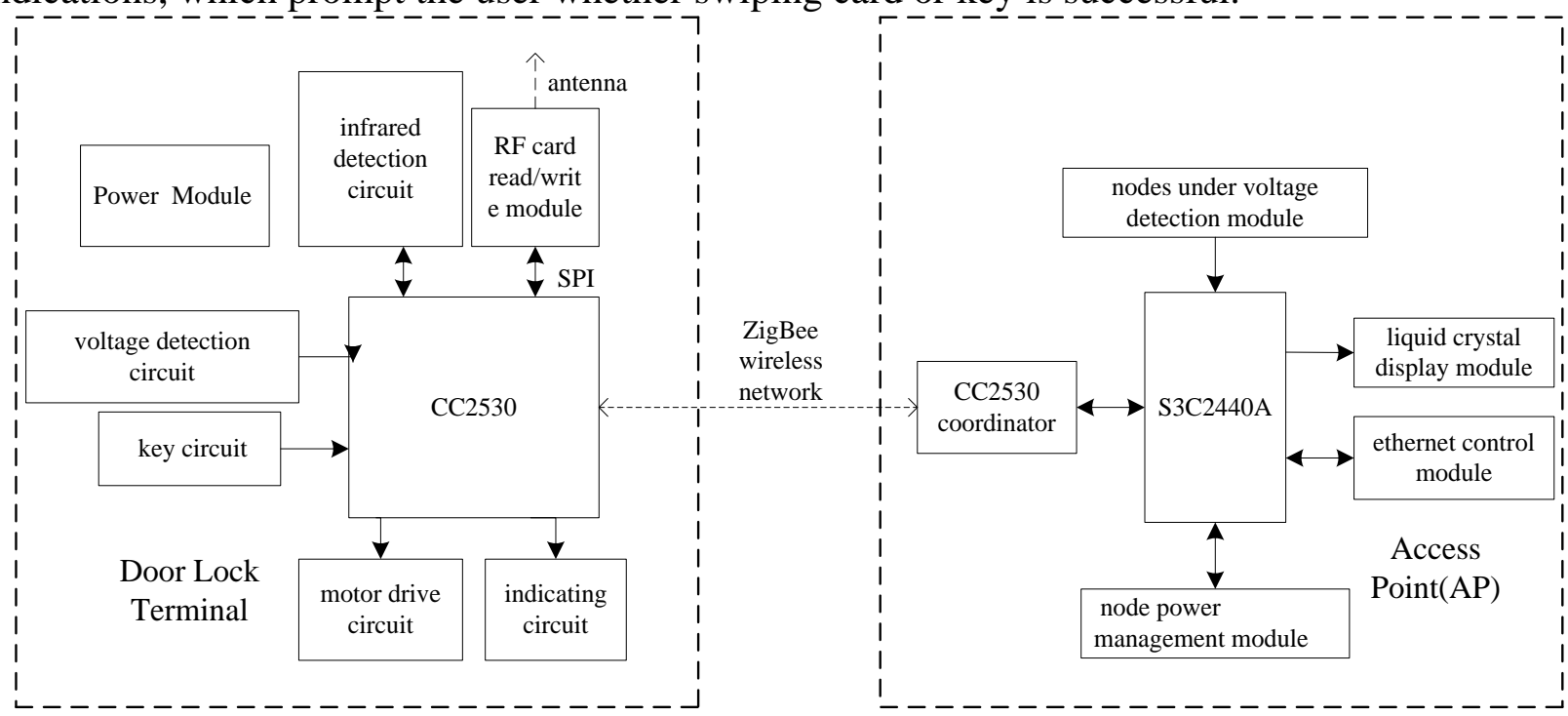

Fig.1. System overall structure

Due to the larger hotels now, in order to enhance the reliability of the network and reduce the message delay, mesh network topology is used between coordinator, router and terminal. The network is set up by the coordinator, when the terminal accesses network, it would send a request to the coordinator. Then the coordinator will assign a 16-bit network address to the terminal.

\section{Hardware design}

The system uses ZigBee communication between the Door Lock Terminal and the AP. ZigBee protocol is a short-range wireless sensor networks and control protocol. Its advantages of small amount of data communication, low complexity, power and cost become the best choice of wireless network transmission protocol, and it is suitable for battery-powered systems. The MCU of door terminal is CC2530. Meanwhile, CC2530 is also the basis for ZigBee communication. CC2530 integrates single-chip, ADC and wireless communication module in a body. It supports the latest ZigBee protocol and IEEE802.15.4, and has virtues of high performance, low price and low power consumption.

\section{The circuit of Reader card and Antenna}

The card reader will receive the card information through the antenna, and read the information transmitted to CC2530. By using the chip of NXP Company MFRC522, the system can get the card information and undertake communication with the MIFARE card, which is being used popular. MFRC522 has advantages of small volume and low power consumption. MFRC522 will transmit data with CC2530 through four wires in the form of SPI. CC2530 exchange the information with MFRC522 by the MISO mouth.

While the MFRC522 is communicating with the MIFARE, the reader writer device sends electromagnetic wave to MIFARE card with a set of the frequency of $13.56 \mathrm{MHz}$ through antenna. 
The antenna coil is a resonance frequency of $13.56 \mathrm{MHz}$ LC circuit, and the resonance frequency equation is as follows:

$$
f=\frac{1}{2 \pi \sqrt{L C}}
$$

$\mathrm{L}, \mathrm{C}$, respectively of the equivalent inductance of the antenna and the equivalent capacitance.

Inside the MIFARE card is a LC series resonance circuit and its frequency is also $13.56 \mathrm{MHz}$. Through the stimulus of electromagnetic wave, LC resonance circuit produces resonance. When LC resonance circuit is reaching a certain power, its internal capacitance can be used as power supply for other circuit inside card working voltage, launch the data in the card, the communication of the MFRC522 with MIFARE card is completed. Figure 2 is MFRC522 circuitry, including antenna and its matching circuit.

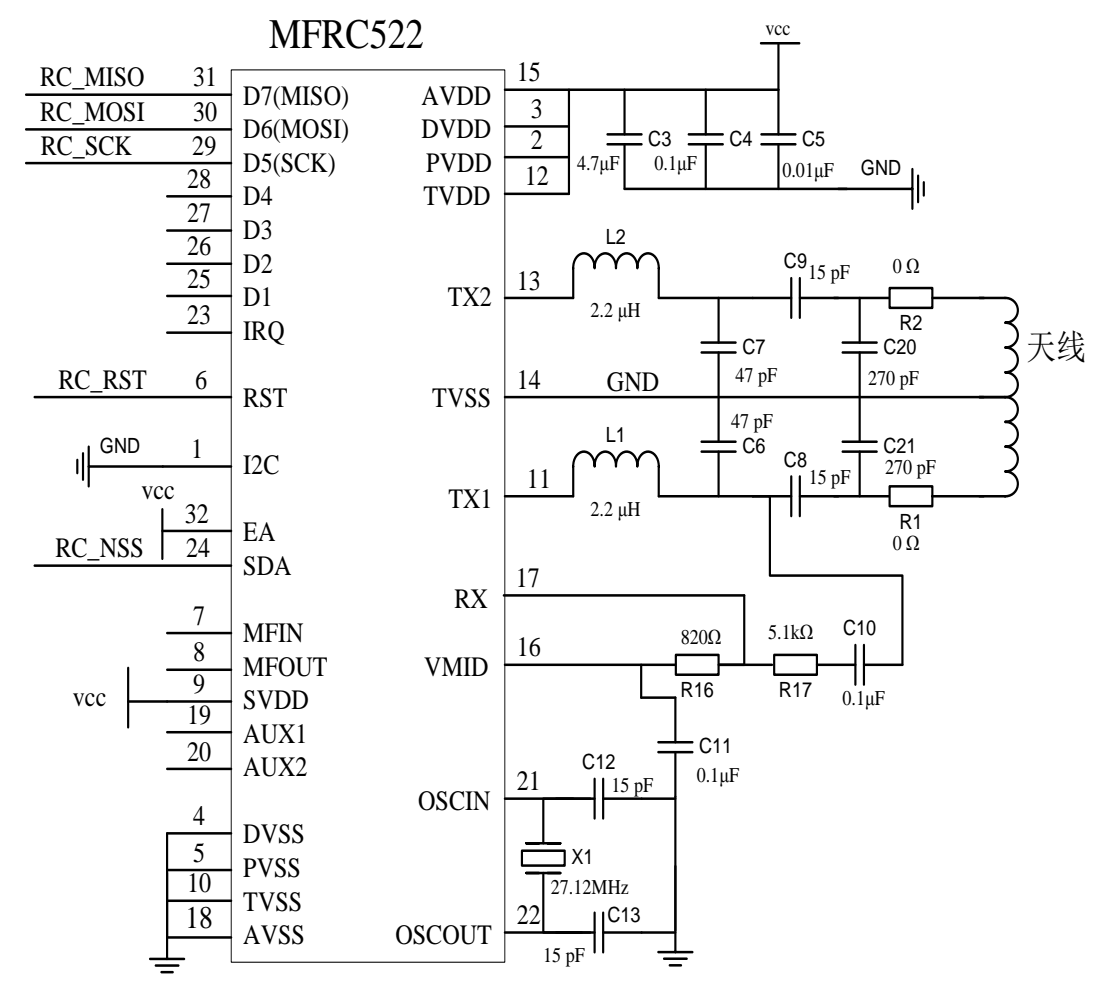

Fig.2. MFRC522 and antenna drive circuit diagram

\section{Power module and voltage detection circuit}

Power module for the system provides stable voltage. Power supply module and voltage detection circuit diagram is shown in figure 3 (a), chip uses ME6209A33. In order to ensure the stability of the system, when VCC is below the threshold voltage, system alarms. CC2530 has an internal ADC, can realize conversion of analog voltage to the digital voltage . PD0 connects to CC2530 I/O pins which has ADC function, R1 and R2 form a voltage divider circuit. The threshold voltage is $0.8 \mathrm{~V}$.

\section{Infrared detection circuit}

Infrared detection circuit is used for detecting whether there is a card. Only when a card existed, card reader starts, it can avoid high power loss due to the card reader working all the time. Door Lock Terminal emits an infrared ray every 0.5 seconds. When a card closed to door lock, LEDR will receive the reflected infrared ray. As shown in figure 3 (b), LEDR conducts at the moment, the voltage of 2 pin of the operational amplifier changes from $3.3 \mathrm{~V}$ to $0 \mathrm{~V}$, the level of PD1 changes from 0 to 1, and CC2530 starts card reader. 

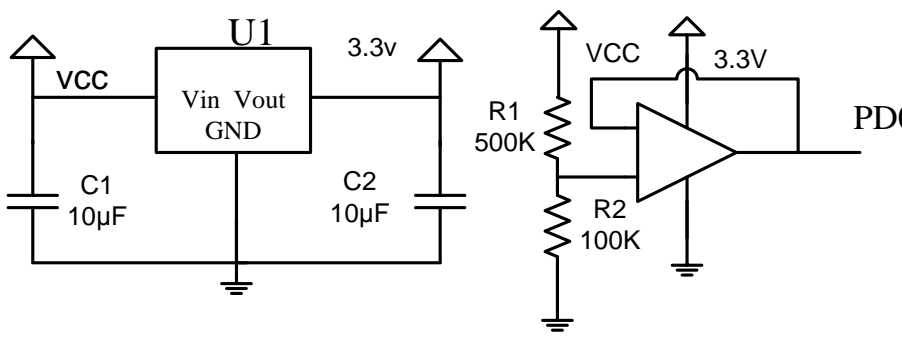

(a) Power module and voltage detection circuit

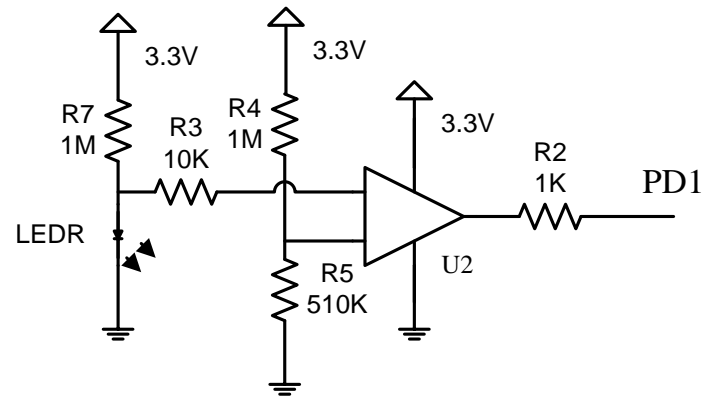

(b) Infrared detection circuit diagram

Fig.1. The hardware circuit diagram

\section{Conclusion}

The system designed in this scheme adopts the way that combines low power high-performance CC2530 with low power small volume card reader chip MFRC522, which further to reduce the system power and volume, to guarantee the Door Lock Terminal running efficiency, durability and stability. The system uses ZigBee communication between the Door Lock Terminal and the AP. This reduces the cost of system communication and operation, and has the advantages of low cost, convenient installation, strong expansibility. The system adds key modules to facilitate customer service, and improve the management efficiency. The system is suitable for the large hotel. Tests have shown that 4 batteries can be used for access control terminal more than half a year. The system can also be extended to intelligent management system as room equipment management system, which makes management of the hotel more intelligent.

\section{References}

[1] Ruohao Zhao, Qinruo Wang, Weixiong Chen.ZigBee technology in the application of the entrance guard system[J].The China science and technology information,2007,21(3):82-85.

[2] Zhu Ming, Junxing Ren, Zhao Xia. Entrance guard system based on Zigbee design [J]. Computer knowledge and technology, 2012, 8(29):6948-6951.

[3] Li Xin. Hotel management system introduced [J]. Journal of intelligent building and city information, 2008, 3(5):34-36.

[4] Zhiwen Wang , Chen Junwu, HongHui. Lab entrance guard system for an open management [J]. Journal of laboratory research and exploration, 2009, 28(6):60-63.

[5] Guoqiang Peng . New five-star hotel intelligent system design need to be aware of the problem [J]. Journal of technology, 2010,116(2):61-64.

[6] Jiahu Guo. Hotel management system and its operation and management methods: China, 201410004285.6 [P]. 2014-04-16.

[7] Edgar H,Callawy J,Callaway E H.Wireless sensor net-works:Architectures and Protocols[M]. New York:Auerbach Pubication,2003:260-300.

[8] Wenqing Chen. The entrance guard system based on ZigBee technology design [J]. Journal of luoyang institute of technology (natural science edition), 2013, 23(4):40-43.

[9] Junxiang Huang, Weiqing Tao. Base on the RFID reader module MFRC522 design [J]. Micro computer and application, 2010, 29(22):16 to 18. 